\title{
Multi-Agent Fuzzy Logic Controller applied to One Lever Variable Pitch Turboprop Aircraft
}

\author{
Lorenzo Dambrosio* \\ Department of Mechanics, Mathematics and Management, Politecnico di Bari, Via Orabona 4, 70125 \\ Bari, Italy
}

\begin{abstract}
Modern aircraft turboprop engines are generally managed by pilots by using the well-established two-levers approach which allows the pilot to control independently the engine thrust (power lever) and the propeller rotational speed (condition lever). On the other hand, the twolevers governing system presents several disadvantages in terms of pilot training, fuel consumption and aircraft maintenance. The one-lever approach tries to solve such drawbacks regulating at the same time the engine power and the propeller pitch. In the present work a Multi-Agent Fuzzy Logic control algorithm has been proposed to implement the one-lever approach for the control of the variable pitch turboprop aircraft propulsion system. The whole aircraft ecosystem has been developed in Simulink ${ }^{\circledR}$ framework to verify the feasibility and performance of the proposed one-lever approach.
\end{abstract}

\section{Introduction}

The way an aircraft propulsion system delivers the thrust has a significant impact on the aircraft flight dynamics. This aspect, in turn, affects the kind of mission the aircraft can perform. All the propulsion systems produce thrust relying on the momentum conservation principle [1]. Nevertheless, the momentum variation of the aircraft engine outlet gases, necessary to provide a given thrust level, can be generated operating either a substantial speed change on a small gas flow rate or a moderate speed change on a significant gas flow rate. The turbojet propulsion systems employ the former approach obtaining the optimal values of efficiency and thrust at high aircraft Velocity True Air Speed (VTAS); of course, such a characteristic is especially appreciated for military applications where high aircraft VTAS is required: fast military fighter planes are equipped with these kinds of propulsion system [2]

On the contrary, the second solution is essentially adopted by aircrafts using turboprop propulsion. Consequently, the best flight conditions of these aircrafts are achieved in low VTAS conditions. Specifically, those aircraft equipped with variable pitch turboprop propulsion system are generally heavier than turbojet ones with comparable engine power output, but, on the other hand, they offer a quite large maximum take-off weight. In addition, such turboprop-based aircrafts yield the best performance in terms of efficiency and thrust in subsonic conditions (takeoff and climb phases). These features make them quite suitable for cargo applications (high payload at average speed). As positive consequences:

${ }^{*}$ Corresponding author: lorenzo.dambrosio@poliba.it 
- $\quad$ since the turboprop aircrafts have a large propeller air mass flow rate and require a small gases acceleration, the thrust-specific fuel consumption of is smaller than that of turbojet aircrafts one.

- due to their high level of thrust, the variable pitch turboprop aircrafts are able to take off and to land on shorter runways than is normally required turbojet-based aircrafts.

- the maintenance costs of the propeller-based propulsion systems are lower with respect to turbojet ones [3].

Historically, in order to allow a rapid takeoff, the first propeller-based propulsion systems adopted fixed pitch propellers featured by a small angle of attack. Nevertheless, such a choice limited the maximum aircraft velocity during the cruise phase. To overcome such a drawback, keeping a high thrust value at low speed (rapid take-off), the variable pitch propellers have been introduced [4]. However, the variable pitch turboprop propulsion system requires a governing unit, called Constant-Speed Propeller, constituted by two independent control inputs (two-levers), to regulate individually the gas turbine output power (power lever) and the propeller rotational speed (condition lever) [5]. These two levers have to be maneuvered by the pilot who, not only has to take care of the aircraft navigation system, but he has also to determine the most appropriate values of the engine output power (power lever) and propeller rotational speed (condition lever) in order to ensure the propeller maximum efficiency condition: thus, the pilot expertise has a significant impact on the cruise fuel consumption. In addition, since the two-levers propulsion management system results more complex than the single-lever one, the aircraft maintenance and the pilot training costs are higher with respect to those of a fixed pitch propeller aircraft.

For these reasons, a great interest has lately emerged towards the one-lever approach to manage the variable pitch propellers propulsion system. In the present work a Multi-Agent System $(M A S)$, featured by Fuzzy Logic Agents, has been implemented to control the onelever management system of the aircraft propulsion [6]. In particular, the MAS algorithm has been applied in Multi Input Single Output mode, where the pitch propeller angle has been determined as a sum of all the agents' contributions. Specifically, the agents included in the proposed MAS are based on a fuzzy logic scheme. Such a choice represents a good trade-off between agents' flexibility, thanks to the internal fuzzy logic framework of each agent and the overall low complexity of the MAS scheme. The aim of the one-lever management system is to optimize the propellers efficiency which translates into a reduction of both fuel consumption and engine maintenance operations.

\section{Variable pitch turboprop aircraft model}

In order to validate the proposed the $M A S$ scheme for the management of the one-lever aircraft propulsion system, the mathematical model of each aircraft component has been built by using Simulink ${ }^{\circledR}$ developing environment which allows an object-oriented approach. As a result, the Simulink ${ }^{\circledR}$ software package provided the simulation of the whole aircraft ecosystem which determines with an adequate level of accuracy the aircraft flight dynamics and the aircraft aerodynamic interactions with the external environment [7]. 


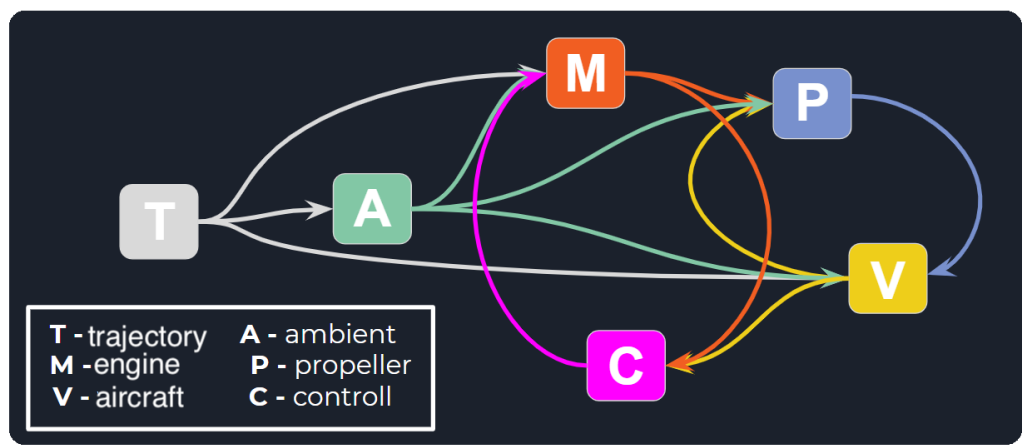

Fig. 1. Mathematical model logic scheme in Simulink ${ }^{\circledR}$ framework.

Figure 1 illustrates the conceptual modules architectures of the turboprop aircraft ecosystem: specifically, the module $\mathbf{T}$ provide the aircraft trajectory, whereas the module $\mathbf{V}$ takes into account the aircraft interactions with the external environment. The engine is constituted by a co-axial twin shaft gas turbine (module $\mathbf{M}$ ) whose low pressure shaft drives the propeller (module P). Finally, the module A determines the environment conditions that affects the engine working conditions whereas the module $\mathbf{C}$ represents the Multi-Agent Scheme that regulates the one-lever management system of the aircraft propulsion. For validation purpose, the aircraft system model outputs have been compared with a particular flight design condition (table 1), specified in [7].

Table 1. Flight design conditions.

\begin{tabular}{|c|c|c|c|}
\hline Flight Condition & Values & Flight Condition & Values \\
\hline Absolute Flight speed $[\mathrm{m} / \mathrm{s}]$ & 34.01 & Compressor pressure ratio & 9.40 \\
\hline Altitude $[\mathrm{m}]$ & 0 & Air mass flow rate $[\mathrm{kg} / \mathrm{s}]$ & 4.18 \\
\hline Ambient air temperature $[K]$ & 288.15 & Compressor thermal efficiency & 0.77 \\
\hline Ambient air pressure $[\mathrm{bar}]$ & 1.013 & High pressure turbine speed $[\mathrm{rpm}]$ & 37500 \\
\hline
\end{tabular}

Furthermore, the engine model had been implemented considering the dynamic mechanical equilibrium of both the high- and low-pressure shafts, whilst the compressor and propeller working point has been determined thanks to their respective maps. Finally, in order to complete the whole aircraft ecosystem in Simulink ${ }^{\circledR}$ environment, the aircraft flight dynamic equilibrium has been considered in the vertical plane. In ref. [7] a complete description of the mathematical model implemented in Simulink ${ }^{\circledR}$ framework, including the propeller and gearbox model is reported.

\section{Multi Agent System}

A Multi Agent System technique offers several potential advantages with respect the single-agent approach such as $[8,9]$. It can

- decompose a problem, allocate subtasks to agents, and synthesize partial results,

- consider a distributed perceptive information of the environment,

- provide a decentralized management granting an efficient coordination mechanism among agents,

- tdesign agents to properly react to the actions, plans, and knowledge of other agents. 
An agent can be viewed as a unit that perceives its environment through input signal and acts upon that environment through output signal, according to its designed task. The degree of success of an agent is generally assessed by the user (agent designer) and reflects the assigned task to that particular the agent. This means that an agent has to consider a decisionmaking problem choosing an action, $\alpha_{t}$, according to the current environment perception, $o_{t}$, and to the past history of perceptions, $o_{\tau}$, and actions, $\alpha_{\tau}$ :

$$
\pi\left(o_{1}, \alpha_{1}, o_{2}, \alpha_{2}, \cdots, o_{t-1}, \alpha_{t-1}, o_{t}\right)=\alpha_{t}
$$

where $\pi$ is called policy function. However, since the complete perception action pairs history can require large amount of memory and computational effort, to overcome such a computational complexity, simpler policies may be used. The so-called reactive or memoryless policy represent a particular simple policy which assumes the following form:

$$
\pi\left(o_{t}\right)=\alpha_{t}
$$

In the present work, the proposed $M A S$ is constituted by agents using such a policy function: such agents are called reflex agents. Of course, the definition of the policy function that performs the mapping between perception and action play a key role. For the present proposed $M A S$, a fuzzy logic system has been employed as policy function [6]. The reasons for such a choice lie on the two following aspects:

- $\quad$ each agent is featured with an adequate level of flexibility, thanks to the fuzzy logic policy function;

- the internal fuzzy logic structure of each agent presents a low level of complexity since each agent has not to face the entire problem.

From the fuzzy logic system point of view, the perception and the corresponding action represent the input and the output of the fuzzy logic system. Moreover, as far as the agents' fuzzy policy function is concerned, the Simulink ${ }^{\circledR}$ Fuzzy Logic Toolbox has been used to build up the Fuzzy Logic System. Specifically, the membership functions of both the rules antecedent and consequent have been chosen to be triangular; the inference engine utilizes a product inference for the rule implication. Finally, the fuzzification has been carried out by using of a singleton fuzzifier, whereas the defuzzification processes have been featured by a height defuzzifier.

\section{Agents Description}

In the present work, the $M A S$ control scheme has been applied in multi input single output mode to manage the control the one-lever variable pitch turboprop propulsion system. The controlled variable is represented by the propeller efficiency, whereas the propeller pitch angle has been considered as control variables. The proposed MAS is composed by four agents and a brief description of them will be outlined.

From Ref. [7], where the same problem of the one-lever variable pitch turboprop propulsion was solved adopting a model reference approach, it has been possible to select a finite number of working point to build a fuzzy base of rules which achieves a mapping between the flight conditions status and the propeller pitch angle. Such a fuzzy logic system constituted the policy function of the first designed agent, called AGENT1. The perception of $A G E N T 1$, or in other words, the input vector of fuzzy logic system, is defined by the flight altitude, the VTAS, and the fuel flow rate. Few considerations are in order:

- AGENT1, according to the flight conditions, provides the baseline of the propeller pitch angle control variable.

- AGENT1 operates on an open-loop scheme, and its policy function has been designed thanks to available data [7] and, for this reason can be updated and improved. 
- Since AGENT1 deliver the baseline of the propeller pitch angle, the efficiency of the one-lever turboprop propulsion system is lower with respect to that obtained in Ref. [7].

- The fuzzy base of rules of AGENT1 is constituted by nine rules for each maneuver.

The action of the second agent, called AGENT2, represents a second contribution to the propeller pitch angle in order to improve the efficiency of the one-lever turboprop propulsion system. It takes as perception vector the optimal value (max efficiency condition) [7] of the propeller airfoil angle of attack and the Advance Ratio $J$, defined as:

$$
J=\frac{V}{n D}
$$

where $n$ represent the propeller rotational speed, $D$ tip diameter of the propeller and $V$ are the aircraft velocity. The fuzzy base of rules of $A G E N T 2$ has been set up taking into account a finite number of working conditions in which it has been possible to determine the pitch angle correction. Similarly to AGENT1, AGENT2 evaluates its action on the basis of flight conditions and propeller max efficiency condition by using an open-loop scheme.

The third agent, $A G E N T 3$, also provides an additional contribution to the propeller pitch angle in order to reduce the difference between the propeller airfoil angle of attack and its optimal value (max efficiency condition) [7], but this time it adopts a closed-loop scheme. Specifically, the AGENT3 performs an integral correction as follows:

$$
\Delta \beta_{i}=K_{i} \int_{0}^{t} e_{\alpha} d \tau,
$$

where $\Delta \beta_{i}$ represents the integral correction to the propeller pitch angle, $K_{i}$ is integral gain, whereas $e_{a}$ indicates the difference between the propeller airfoil angle of attack and its optimal value [7]. Moreover, AGENT3, performs a fuzzy selection of the $K_{i}$ parameter according to $e_{a}$ values. In other words, thanks to a fuzzy base of rule constituted by five rules, $A G E N T 3$ considers small $K_{i}$ values when $e_{a}$ is large and, vice versa, large integral gain when the error of the propeller airfoil angle of attack is small. The basic idea behind such scheme relies on the following consideration: the integral contribution can effectively drive to zero angle of attack control error when $e_{a}$ is quite small. Otherwise, it will produce an undesired oscillating behavior. Therefore, AGENT1 and AGENT2 will provide main the control action when $e_{a}$ is large whereas, for small $e_{a}, A G E N T 3$ will drive to zero the angle of attack error.

Since the variable pitch turboprop propulsion system is based on so-called ConstantSpeed Propeller approach, a fourth agent, called AGENT4, has been proposed in order to take care of rotational speed variation of the propeller shaft. Also, this agent presents a fuzzy internal structure with propeller rotational speed error and flight altitude as inputs (perception) and the propeller pitch angle correction as output (action). Since the AGENT4 gives a contribution only when a significant variation in propeller rotational speed occurs, its action is zero during the normal flight maneuvers, like those described in the result section. For this reason, AGENT4 plays, in some sense, the role of flight conditions diagnostic unit.

\section{Results}

In this section the results concerning the application of the proposed $M A S$ scheme for the control of the one-lever turboprop propulsion system will be presented. Two different flight maneuvers have been considered: the Simple Takeoff and the Simple Descent. It would be interesting to compare these results with those obtained performing such maneuvers by using the traditional two-lever system; unfortunately, these data are not easily available. Nevertheless, some concluding remarks can still be made. In order to perform such maneuvers, the aircraft SAAB 340 has been selected as study case. This is due not only to 
the features and mission of this aircraft that well correspond to the modeled turboprop propulsive system but also because the characteristics and main flight data of this aircraft are easily available. In this way, the block Trajectory can provide realistic trajectory data.

The Simple Takeoff maneuver neglects the runway acceleration and takes the initial aircraft velocity equal to $34.04 \mathrm{~m} / \mathrm{s}$ reaching a final level flight condition at the altitude of $2000 \mathrm{~m}$, as illustrated in Fig. 2. Figure 3 reports the required fuel mass flow rate for the medium load conditions $(10483 \mathrm{~kg})$. It is worth mentioning that for the two-lever approach, this maneuver would be carried out with the maximum power demand or, in other words, with the power lever set in takeoff position. This means that, during the Simple Takeoff maneuver the two-lever approach needs the maximum fuel mass flow rate
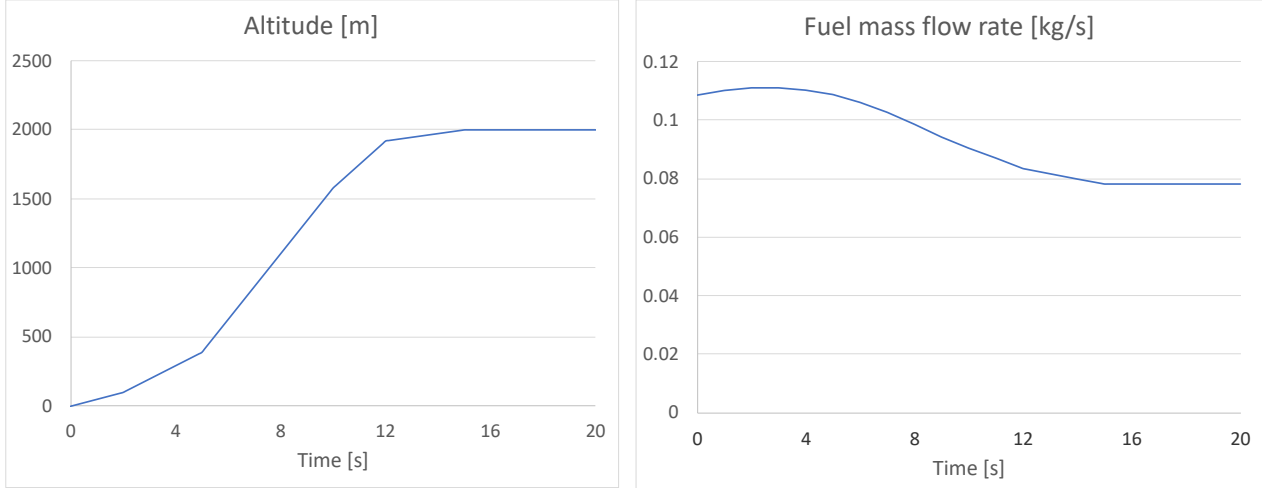

Fig. 2. Simple Takeoff - Altitude vs. Time

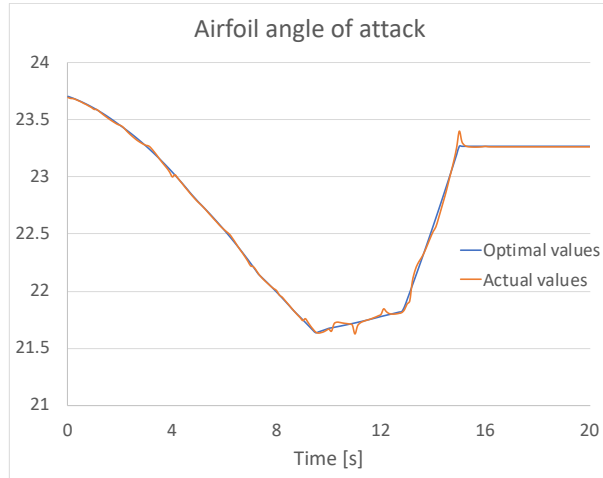

Fig. 3. Simple Takeoff - Fuel Consumption vs. Time

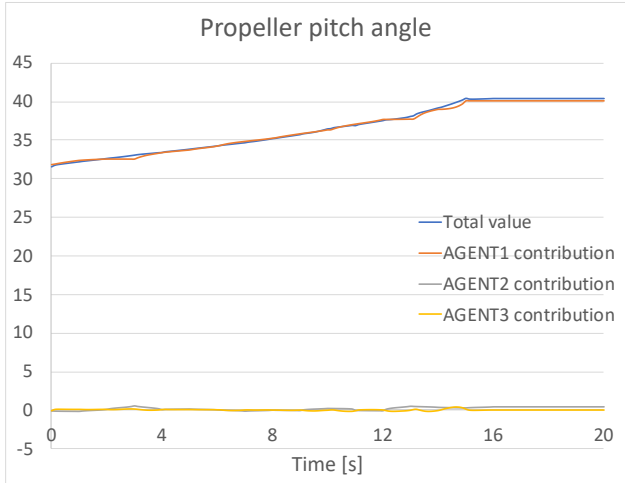

Fig. 4. Simple Takeoff - Propeller airfoil angle of attack vs. Time

Fig. 5. Simple Takeoff - Propeller pitch angle vs. Time

without taking into account the flight conditions and the considered payload. Figure 4 reports the propeller airfoil angle of attack, both optimal and actual values, vs. time, where the optimal angle of attack values have been derived from [7]. It is noticeable that the proposed $M A S$ makes sure that the propeller airfoil angle of attack tracks the corresponding optimal values during the entire maneuver; in particular, only during transient periods the airfoil angle of attack control error is barely noticeable, whereas in steady state condition there is no control error. Figure 5 illustrates the total value of the propeller pitch angle and the contributions of each single agent. It is quite evident that AGENT1 provides the pitch angle baseline, whilst $A G E N T 2$ and AGENT3 take care of the control error accuracy (low efficiency losses). In this context, Fig. 6 shows the propeller efficiency vs time and from a quick glance at this figure, it is possible to state that the proposed $M A S$ control scheme applied to the onelever management propulsion system is able to effectively maintain a high propeller 
efficiency value during the entire Simple Takeoff maneuver. This aspect confirms that the proposed methodology offers a more efficient control of the propulsive system.
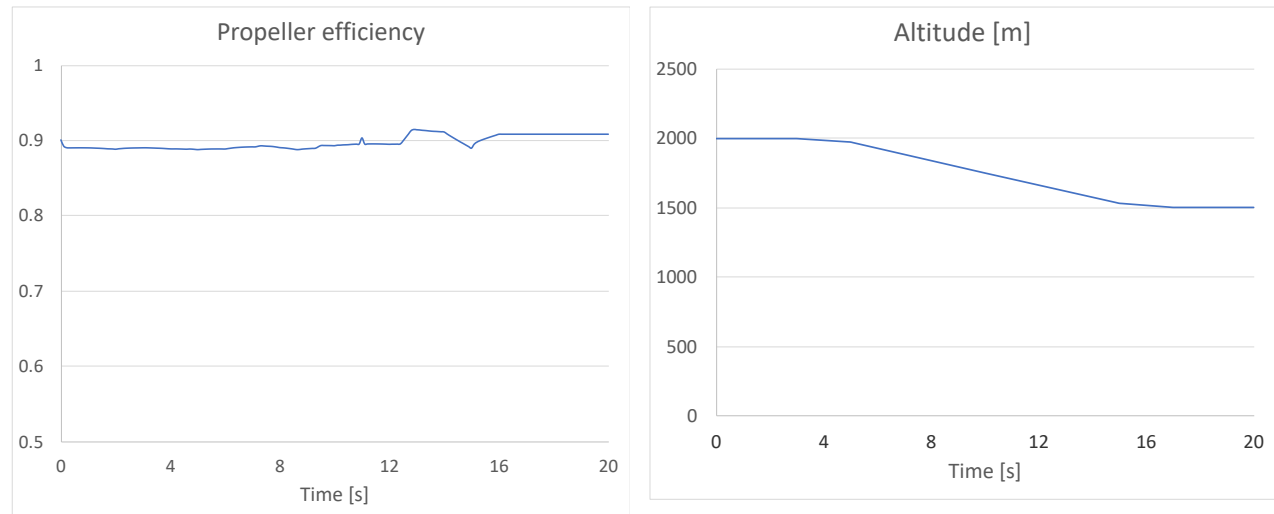

Fig. 6. Simple Takeoff - Propeller efficiency of attack vs. Time

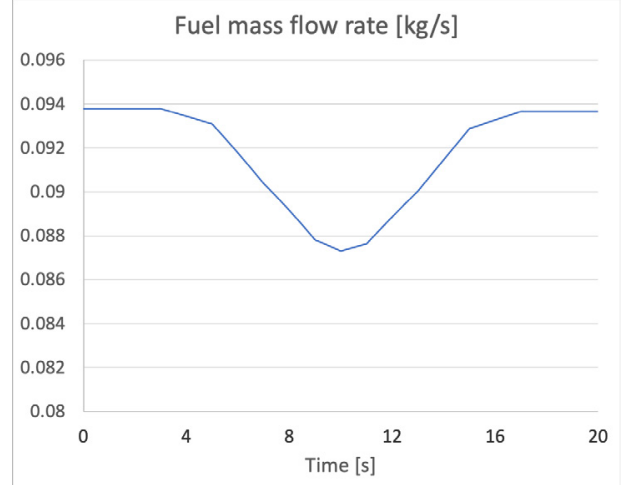

Fig. 8. Simple Descent - Fuel Consumption vs. Time

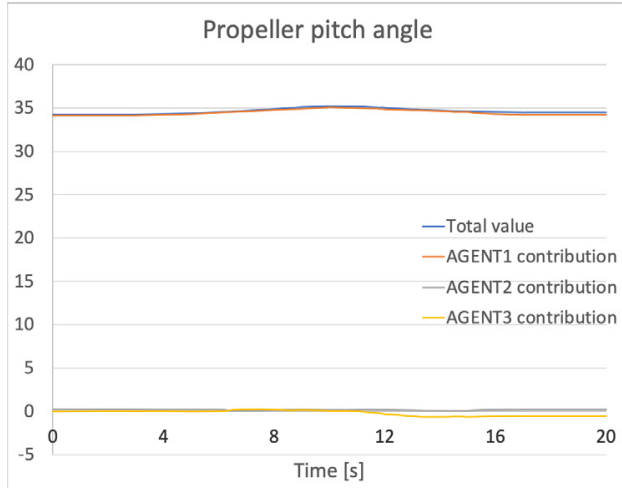

Fig. 10. Simple Descent - Altitude vs. Time

Fig. 7. Simple Descent - Altitude vs. Time

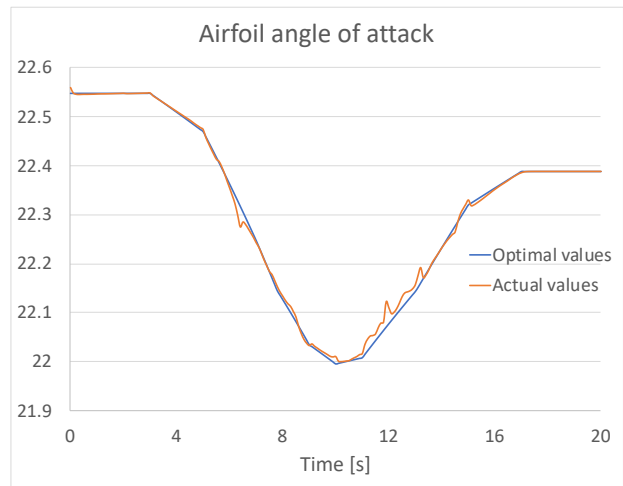

Fig. 9. Simple Descent - Propeller airfoil angle of attack vs. Time

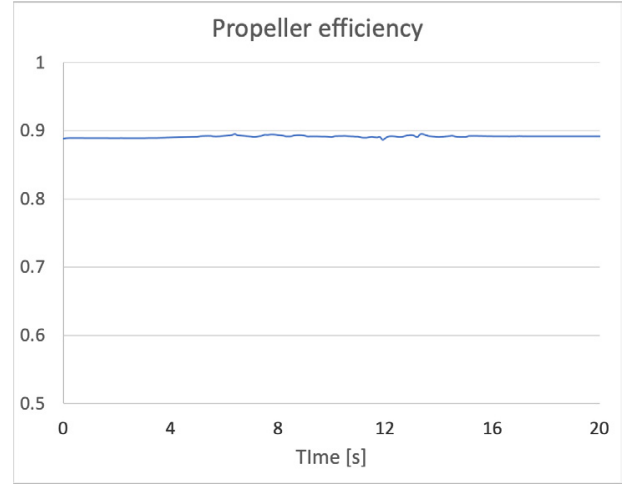

Fig. 12. Simple Descent - Aircraft velocity vs.

Time

The second maneuver is the Simple Descent and takes place in a vertical plane. Figure 7 illustrates the altitude variation of the Simple Descent maneuver, whereas Fig. 8 depicts the fuel mass flow rate for the medium payload condition. Even if no two-levers turboprop propulsion system data are available, however it is possible to observer that, considering the two-levers approach, different pilots would perform the same flight maneuver (Simple Descent) with different performance in terms of airfoil efficiency, propeller promptness 
response and control effort. This consideration allows to remark that the proposed one-lever $M A S$ controller is not an autopilot, but it helps the pilot during the maneuver providing the requested power in maximum propeller efficiency condition. Likewise to the previous case, Figs. 9 and 10 depict the propeller airfoil angle of attack, both optimal (Ref. [7]) and actual values, and all agents' contributions to the propeller pitch angle, respectively. Again, it is possible to observe a good control tracking of the angle of attack respect to the optimal reference value during the entire maneuver. As far as the propeller pitch angle is concerned, even in the Simple Descent, AGENT1 output represents the baseline value whereas, AGENT2 and $A G E N T 3$ increase control accuracy and the propeller efficiency. Then, Fig. 11 shows the high efficiency level during the entire Simple Descent maneuver.

\section{Conclusions}

The present work deals with a new control strategy approach applied to the propulsion system of a variable pitch turboprop aircraft. Generally, the aircrafts equipped the variable pitch turboprop propulsion system employ the well settled solution composed by a two-lever governing system to regulate both the engine output power and the propeller thrust. Nevertheless, the two-lever system requires, on one hand, a greater pilot expertise and, on the other hand, it implies higher costs in terms of fuel consumption, aircraft maintenance and pilot training. To solve these drawbacks, a Multi Agent System based the one-lever solution for the control of the variable pitch turboprop propulsion system has been proposed.

The MAS technique offers several advantages with respect the single-agent approach: it decomposes the entire problem, provides a decentralized management and considers a distributed architecture of the control unit. In order to validate the viability and effectiveness of the one-lever approach for the management of the variable pitch turboprop propulsion system, at first the whole aircraft ecosystem has been developed in Simulink ${ }^{\circledR}$ framework and afterwards the one-lever Multi-Agent Fuzzy Logic control algorithm has been applied.

All the results have been remarkably encouraging, not only showing better efficiency and fuel consumption, but also pointing out the robustness, the accuracy and the collaborative nature of the proposed $M A S$ control scheme applied to the one-lever management propulsion.

\section{References}

1. U. Ghezzi, Motori per Aeromobili, politecnica, Maggioli Editore, ISBN 8838741867 (2008)

2. R. Storm, M. Skor, L.D. koch, T. Benson, C. Galica, Pushing the Envelope: A NASA Guide to Engines, NASA, (2015)

3. A. Hasselrot, B. Montgomerie, FOI - Swedish Defence Research Agency pp. 1-66 (2005)

4. G. Zizzi, ed., Storia ed evoluzione della propulsione aerea, Stem Mucchi Editore, Modena, Italy, ISBN B000Z9SPDU (1977)

5. J.L. Musgrave, Single-Lever Power Control for General Aviation Aircraft Promises Improved Efficiency and Simplified Pilot Controls, NASA, (1997)

6. L. Dambrosio, M. Mastrovito, S. M. Camporeale ASME. J. Eng. Gas Turbines Power 129(3) 738-745 (2007)

7. G. Gaudio, L. Dambrosio E3S Web Conf. 19711012 (2020)

8. N. Vlassis AI Intelligent Autonomous Systems Informatics Institute. University of Amsterdam, (2003)

9. S. J. Russell and P. Norvig. Artificial intelligence: a modern approach. Prentice Hall, 2nd edition, (2003) 